\title{
Staphylococcus aureus Clinical Isolates: Antibiotic Susceptibility, Molecular Characteristics, and Ability to Form Biofilm
}

\author{
N. Indrawattana, ${ }^{1}$ O. Sungkhachat, ${ }^{1}$ N. Sookrung, ${ }^{2}$ \\ M. Chongsa-nguan, ${ }^{1}$ A. Tungtrongchitr, ${ }^{3}$ S. P. Voravuthikunchai, ${ }^{4}$ \\ T. Kong-ngoen, ${ }^{1}$ H. Kurazono, ${ }^{5}$ and W. Chaicumpa ${ }^{3}$ \\ ${ }^{1}$ Department of Microbiology and Immunology, Faculty of Tropical Medicine, Mahidol University, Bangkok 10400, Thailand \\ ${ }^{2}$ Department of Research and Development, Faculty of Medicine Siriraj Hospital, Mahidol University, Bangkok 10700, Thailand \\ ${ }^{3}$ Department of Parasitology, Faculty of Medicine Siriraj Hospital, Mahidol University, Bangkok 10700, Thailand \\ ${ }^{4}$ Natural Products Research Center and Department of Microbiology, Faculty of Science, Prince of Songkla University, \\ Songkhla 90112, Thailand \\ ${ }^{5}$ Department of Applied Veterinary Medicine and Public Health, Obihiro University of Agriculture and Veterinary Medicine, \\ Inada-cho, Obihiro, Hokkaido 080 8555, Japan
}

Correspondence should be addressed to W. Chaicumpa; wanpen.cha@mahidol.ac.th

Received 30 May 2013; Accepted 24 July 2013

Academic Editor: Sou-ichi Makino

Copyright (c) 2013 N. Indrawattana et al. This is an open access article distributed under the Creative Commons Attribution License, which permits unrestricted use, distribution, and reproduction in any medium, provided the original work is properly cited.

\begin{abstract}
Periodic monitoring of Staphylococcus aureus characteristics in a locality is imperative as their drug-resistant variants cause treatment problem. In this study, antibiograms, prevalence of toxin genes (sea-see, seg-ser, seu, tsst-1, eta, etb, and etd), PFGE types, accessory gene regulator (agr) groups, and ability to form biofilm of $92 \mathrm{~S}$. aureus Thailand clinical isolates were investigated. They were classified into 10 drug groups: groups 1-7 (56 isolates) were methicillin resistant (MRSA) and 8-10 (36 isolates) were methicillin sensitive (MSSA). One isolate did not have any toxin gene, 4 isolates carried one toxin gene (seq), and 87 isolates had two or more toxin genes. No isolate had see, etb, or tsst-1; six isolates had eta or etd. Combined seg-sei-sem-sen-seo of the highly prevalent egc locus was $26.1 \%$. The $s e b, s e c$, sel, seu, and eta associated significantly with MSSA; sek was more in MRSA. The sek-seq association was $52.17 \%$ while combined sed-sej was not found. Twenty-three PFGE types were revealed, no association of toxin genes with PFGE types. All four agr groups were present; agr group 1 was predominant (58.70\%) but agr group 2 strains carried more toxin genes and were more frequent toxin producers. Biofilm formation was found in $72.83 \%$ of the isolates but there was no association with antibiograms. This study provides insight information on molecular and phenotypic markers of Thailand S. aureus clinical isolates which should be useful for future active surveillance that aimed to control a spread of existing antimicrobial resistant bacteria and early recognition of a newly emerged variant.
\end{abstract}

\section{Introduction}

Staphylococcus aureus, a gram positive coccal bacterium, is either commensal that colonizes healthy nasal mucosa [1] or pathogen of humans. As a pathogen, the bacteria cause a variety of community and hospital acquired diseases including skin abscess [2], food poisoning [3], pneumonitis [4], sepsis [5], and toxic shock syndrome [6]. This bacterium produces several virulent factors including adhesins (colonization factors), toxic proteins/enzymes (e.g., DNase for bacterial spread, coagulase, and catalase for host immunity evasion) and exotoxins including exfoliative toxins (ExTs), staphylococcal enterotoxins (SEs), and toxic shock syndrome toxin-1 (TSST-1). Patients infected with the ExT producing S. aureus may develop scalded-skin syndrome [7]. The SEs and TSST-1, besides causing food poisoning, are also superantigens (SAg) that can stimulate a relatively large fraction of peripheral blood $\mathrm{T}$ cells to release massive amounts of proinflammatory 
cytokines and T-cell stimulating factors leading to toxic shock syndrome which may be fatal $[8,9]$. The enterotoxicity and superantigenicity are distinct properties of the toxin molecule [6]. SEs are classified into two types based on their emetic activity in the toxin fed modeled primate. Toxins that induce vomiting in the primate are placed in the classical SE type while those that lack the emetic activity or have not been tested are allocated in the SE-like (SEls) type $[10,11]$. Members of the classical SEs are SEA-SEE and the more recently recognized SEG, SEH, SEI, SER, SES, and SET. The SEls members include SElJ, SElK, SElL, SElM, SElN, SElO, SElP, SElQ, SElU, SElU2 or SEW, and SElV [11]. The staphylococcal enterotoxin F (SEF) which lacks emetic activity but is associated with toxic shock syndrome is presently called toxic shock syndrome toxin-1 (TSST-1) [12]. The SEs and the TSST-1 as well as the bacterial resistance to drugs are encoded by genes on the mobile genetic elements including prophages, plasmids, pathogenicity islands, genomic islands, and antibiotic resistance cassette [13]; thus they are transmitted horizontally rather easily. Expression of S. aureus virulence factors and metabolism of metabolic pathways during growth are coordinated/regulated by a quorumsensing operon named accessory gene regulator (agr) [14, 15]. Based on the amino acid sequence polymorphisms of the agr-encoding autoinducing peptides and their responding receptors, $S$. aureus strains can be divided into four major $a g r$ groups (groups 1-4) [16].

During the last five decades, $S$. aureus clones that resist methicillin (methicillin-resistant $S$. aureus, MRSA) disseminated and caused medical and public health problem worldwide $[17,18]$. These strains are not only resistant to methicillin, but also resistant to all other $\beta$-lactams, such as cephalosporin $[18,19]$. In Thailand, MRSA infections were reported from 23 hospitals from 1988 to 1998 [20, 21]. The proportions of MRSA to MSSA in the northeast, central, and southern regions of the country during the studied period increased from 11 to $23.4 \%, 16$ to $30.5 \%$, and 21 to $30.3 \%$, respectively [22]. Moreover, methicillin-resistant S. aureus with reduced susceptibility to vancomycin was recognized [23]. However, data on genotypic characteristics and other attributes of the $S$. aureus isolates in Thailand are relatively rare. Therefore, this study investigated the prevalence of virulence toxin genes coding for enterotoxins (sea-see, seg-ser, and seu), toxic shock syndrome toxin-1 (tsst-1), and exfoliative toxins (eta, etb, and etd) among $S$. aureus Thailand clinical isolates. Molecular diversity of the isolates regarding their endonuclease-restricted patterns of genomic DNA (PFGE), agr types, and antimicrobial susceptibility as well as their ability to produce biofilm were also investigated.

\section{Materials and Methods}

2.1. Bacterial Strains. Ninety-two strains of S. aureus isolated from clinical specimens were obtained from three hospitals. They were 43 strains (S1-S43) isolated in 2007 from patients of Prince of Songkla University Teaching Hospital and kept at the Department of Microbiology, Faculty of Science, Prince of Songkla University, Songkhla province, southern Thailand; 36 strains (P1-P36) from the patients of Prasat Neurological
Institute, Bangkok, in 2010, and 13 strains (T1-T13) isolated in 2010 from patients of the Hospital for Tropical Diseases, Faculty of Tropical Medicine, Mahidol University, Bangkok, Thailand. The bacteria were reconfirmed by Gram staining, biochemical testing (catalase, coagulase, and DNase), and mannitol fermentation. Their ability to produce protein A was detected by agglutination assay.

2.2. Antimicrobial Susceptibility Testing. Disc diffusion method was used for antimicrobial susceptibility testing of the $S$. aureus isolates which was done according to CLSI guidelines [24]. Antibiotic discs were cefoxitin, ciprofloxacin, clindamycin, erythromycin, gentamycin, oxacillin, penicillin $\mathrm{G}$, rifampin, tetracycline, sulfamethoxazole plus trimethoprim, and teicoplanin (Oxoid, UK). Cefoxitin disc $(30 \mu \mathrm{g})$ and oxacillin disc $(1 \mu \mathrm{g})$ were used for detecting methicillinresistant isolates. S. aureus ATCC 25923 was used as control. Reduction of vancomycin susceptibility of the isolates was also determined by observing the minimum inhibitory concentration (MIC) by agar dilution according to the CLSI guidelines [24].

\subsection{Detection of Genes Coding for Staphylococcal Enterotoxins,} TSST-1, and ExTs. Genomic DNA was extracted from each S. aureus isolate by DNA extraction kit (Geneaid, Taiwan) following the protocol for Gram-positive bacteria. Quality of each DNA preparation was assessed by determining the ratio of $\mathrm{OD}_{260 \mathrm{~nm}} / \mathrm{OD}_{280 \mathrm{~nm}}$. Twenty-two virulence genes were amplified including sea-see, seg-ser and seu, tsst-1 and eta, $e t b$ and $e t d$, using specific oligonucleotide primer sequences listed in Table $1[25,26]$. The PCR reaction mixture $(25 \mu \mathrm{L})$ is composed of $1 \mathrm{mM}$ of each primer, $1 \mathrm{x}$ Taq buffer PCR, $0.2 \mathrm{mM}$ dNTP, $2 \mathrm{mM} \mathrm{MgCl}$, 1 unit of Taq DNA polymerase (Fermentas, Germany), and 100 ng of DNA template. The PCR reaction mixture was subjected to the thermal cycles: an initial denaturation of DNA at $95^{\circ} \mathrm{C}$ for $10 \mathrm{~min}$ prior to 35 cycles of denaturation at $95^{\circ} \mathrm{C}$ for $30 \mathrm{sec}, 55^{\circ} \mathrm{C}$ for $30 \mathrm{sec}$, and $72^{\circ} \mathrm{C}$ for $30 \mathrm{sec}$, followed by a final extension of $10 \mathrm{~min}$ at $72^{\circ} \mathrm{C}$ using the Lifecycler (BioRad, USA). The amplified products were analyzed by $1.5 \%$ agarose gel electrophoresis and ethidium bromide staining. The DNA bands were observed under an UV transilluminator (Syngene, England). Control bacteria for the PCR included strains ATCC 19095 (sea, sec, seh, seg, sei, sel, sem, sen, seo, seu, and tst), ATCC 14458 (seb and sek), ATCC 23235 (sed, sej), and ATCC 27664 (see, seq, and sea). For et $a$, $e t b$, and $e t d$, the PCR amplicons were verified by DNA sequencing and the nucleotide sequences were aligned with the staphylococcal eta, etb, and etd sequences of the database (accession numbers: L25372.1, M17348.1, and AB057421.1, resp.).

2.4. Detection of SEs, TSST-1, and ExTs. The bacterial isolates which carried sea, seb, sec and sed; eta and etb; tsst- 1 were tested for their ability to express the respective proteins by the reversed-passive latex agglutination (RPLA) using commercially available kits: SET-RPLA, TST-RPLA, and EXT-RPLA (Denka Seiken, Japan), respectively. Other toxin detections were not done due to lack of available test kits. 
TABLE 1: The primer sequences for amplification of the $S$. aureus enterotoxin genes.

\begin{tabular}{|c|c|c|c|c|}
\hline Target gene & & Primer sequence $5^{\prime} \rightarrow 3^{\prime}$ & $\begin{array}{l}\text { Size of PCR product } \\
\text { (bp) }\end{array}$ & Reference \\
\hline sea & $\begin{array}{l}\text { (F) } \\
(\mathrm{R})\end{array}$ & $\begin{array}{l}\text { GAAAAAAGTCTGAATTGCAGGGAACA } \\
\text { CAAATAAATCGTAATTAACCGAAGGTTC }\end{array}$ & 560 & [26] \\
\hline seb & $\begin{array}{l}(\mathrm{F}) \\
(\mathrm{R})\end{array}$ & $\begin{array}{l}\text { ATTCTATTAAGGACACTAAGTTAGGGA } \\
\text { ATCCCGTTTCATAAGGCGAGT }\end{array}$ & 404 & [26] \\
\hline $\mathrm{sec}$ & $\begin{array}{l}(\mathrm{F}) \\
(\mathrm{R})\end{array}$ & $\begin{array}{l}\text { CTTGTATGTATGGAGGAATAACAAAACATG } \\
\text { CATATCATACCAAAAAGTATTGCCGT }\end{array}$ & 275 & {$[26]$} \\
\hline sed & $\begin{array}{l}(\mathrm{F}) \\
(\mathrm{R})\end{array}$ & $\begin{array}{l}\text { GAATTAAGTAGTACCGCGCTAAATAATATG } \\
\text { GCTGTATTTTTCCTCCGAGAGT }\end{array}$ & 492 & {$[26]$} \\
\hline see & $\begin{array}{l}(\mathrm{F}) \\
(\mathrm{R})\end{array}$ & $\begin{array}{c}\text { CAAAGAAATGCTTTAAGCAATCTTAGGC } \\
\text { CACCTTACCGCCAAAGCTG }\end{array}$ & 482 & [26] \\
\hline seg & $\begin{array}{l}(\mathrm{F}) \\
(\mathrm{R})\end{array}$ & $\begin{array}{l}\text { ACCTGAAAAGCTTCAAGGA } \\
\text { CGCCAACGTAATTCCAC }\end{array}$ & 204 & {$[26]$} \\
\hline seh & $\begin{array}{l}(\mathrm{F}) \\
(\mathrm{R})\end{array}$ & $\begin{array}{l}\text { CAATCACATCATATGCGAAAGCAG } \\
\text { CATCTACCCAAACATTAGCACC }\end{array}$ & 376 & {$[26]$} \\
\hline sei & $\begin{array}{l}(\mathrm{F}) \\
(\mathrm{R})\end{array}$ & $\begin{array}{c}\text { CTYGAATTTTCAACMGGTAC } \\
\text { AGGCAGTCCATCTCCTG-3 }\end{array}$ & 461 & {$[26]$} \\
\hline sej & $\begin{array}{l}(\mathrm{F}) \\
(\mathrm{R})\end{array}$ & $\begin{array}{l}\text { TCAGAACTGTTGTTCCGCTAG } \\
\text { GAATTTTACCAYCAAAGGTAC }\end{array}$ & 138 & {$[26]$} \\
\hline sek & $\begin{array}{l}\text { (F) } \\
\text { (R1) } \\
\text { (R2) }\end{array}$ & $\begin{array}{c}\text { ATGCCAGCGCTCAAGGC } \\
\text { AGATTCATTTGAAAATTGTAGTTGATTAGCT } \\
\text { TGCCAGCGCTCAAGGTG }\end{array}$ & 134 & [26] \\
\hline sel & $\begin{array}{l}(\mathrm{F}) \\
(\mathrm{R})\end{array}$ & $\begin{array}{c}\text { GCGATGTAGGTCCAGGAAAC } \\
\text { CATATATAGTACGAGAGTTAGAACCATA }\end{array}$ & 234 & {$[26]$} \\
\hline sem & $\begin{array}{l}(\mathrm{F}) \\
(\mathrm{R})\end{array}$ & $\begin{array}{l}\text { CTATTAATCTTTGGGTTAATGGAGAAC } \\
\text { TTCAGTTTCGACAGTTTTGTTGTCAT }\end{array}$ & 326 & {$[26]$} \\
\hline sen & $\begin{array}{l}(\mathrm{F}) \\
(\mathrm{R})\end{array}$ & $\begin{array}{l}\text { CGTGGCAATTAGACGAGTC } \\
\text { GATTGATYTTGATGATTATKAG }\end{array}$ & 474 & {$[26]$} \\
\hline seo & $\begin{array}{l}(\mathrm{F}) \\
(\mathrm{R})\end{array}$ & $\begin{array}{l}\text { AGTTTGTGTAAGAAGTCAAGTGTAGA } \\
\text { ATCTTTAAATTCAGCAGATATTCCATCTAAC }\end{array}$ & 180 & [26] \\
\hline sep & $\begin{array}{l}(\mathrm{F}) \\
(\mathrm{R})\end{array}$ & $\begin{array}{l}\text { GAATTGCAGGGAACTGCT } \\
\text { GGCGGTGTCTTTTGAAC }\end{array}$ & 182 & {$[26]$} \\
\hline seq & $\begin{array}{l}(\mathrm{F}) \\
(\mathrm{R})\end{array}$ & $\begin{array}{l}\text { ACCTGAAAAGCTTCAAGGA } \\
\text { CGCCAACGTAATTCCAC }\end{array}$ & 204 & [26] \\
\hline ser & $\begin{array}{l}(\mathrm{F}) \\
(\mathrm{R})\end{array}$ & $\begin{array}{l}\text { AGCGGTAATAGCAGAAAATG } \\
\text { TCTTGTACCGTAACCGTTTT }\end{array}$ & 363 & [26] \\
\hline seu & $\begin{array}{l}(\mathrm{F}) \\
(\mathrm{R})\end{array}$ & $\begin{array}{l}\text { AATGGCTCTAAAATTGATGG } \\
\text { ATTTGATTTCCATCATGCTC }\end{array}$ & 215 & {$[26]$} \\
\hline tst & $\begin{array}{l}(\mathrm{F}) \\
(\mathrm{R})\end{array}$ & $\begin{array}{l}\text { TTCACTATTTGTAAAAGTGTCAGACCCACT } \\
\text { TACTAATGAATTTTTTTATCGTAAGCCCTT }\end{array}$ & 180 & {$[26]$} \\
\hline eta & $\begin{array}{l}(\mathrm{F}) \\
(\mathrm{R})\end{array}$ & $\begin{array}{c}\text { ACTGTAGGAGCTAGTGCATTTGT } \\
\text { TGGATACTTTTGTCTATCTTTTTCATCAAC }\end{array}$ & 190 & [26] \\
\hline$e t b$ & $\begin{array}{l}(\mathrm{F}) \\
(\mathrm{R})\end{array}$ & $\begin{array}{l}\text { CAGATAAAGAGCTTTATACACACATTAC } \\
\text { AGTGAACTTATCTTTCTATTGAAAAACACTC }\end{array}$ & 612 & [25] \\
\hline etd & $\begin{array}{l}(\mathrm{F}) \\
(\mathrm{R})\end{array}$ & $\begin{array}{c}\text { CAAACTATCATGTATCAAGGATGG } \\
\text { CCAGAATTTCCCGACTCAG }\end{array}$ & 358 & [26] \\
\hline
\end{tabular}

2.5. Pulsed-Field Gel Electrophoresis (PFGE). PFGE patterns of chromosomal DNA of all $S$. aureus isolates were determined by digesting each DNA preparation with SmaI. The digested DNA preparations were subjected to electrophoretic separation in a CHEF-DR II system (BioRad, USA) as described previously [27]. DNA fragment patterns were analyzed in the GeneDirectory Application Version 2.01.00 Copyright 2000-2008 Synoptics Ltd. Percent similarities were identified on dendrogram derived from the unweighted pair group method with arithmetic averages (UPGMA) and based on Dice coefficients. Band position tolerance was set at $1.0 \%$. A coefficient similarity of $70 \%$ was selected to define cluster of the PFGE types.

2.6. The Agr Alleles. Genomic DNA of the 92 S. aureus isolates was used as templates for amplification of agr alleles using 
the group specific primers [16]. The common forward (pan) primer: (5' -ATGCACATGGTGCACATGC- $\left.3^{\prime}\right)$ and reversed primers including: agrl $\left(5^{\prime}\right.$-GTCACAAGTACTATAAGCTGCGAT- $\left.3^{\prime}\right)$, agr2 (5' ${ }^{\prime}$-TATTACTAATTGAAAAGTGCCATAGC-3 $\left.{ }^{\prime}\right)$, agr3 ( $5^{\prime}$-GTAATGTAATAGCTTGTATAATAATACCCAG- $3^{\prime}$ ), and agr4 ( $5^{\prime}$-CGATAATGCCGTAATACCCG- $3^{\prime}$ ) were used. These primers allowed amplification of 439-, 572-, 320-, and 657-bp DNA fragments of the agr groups $1-4$, respectively.

2.7. Biofilm Formation. Ability of the S. aureus isolates to form biofilm was determined according to the protocol described previously [28] with modification. Individual bacterial isolates were cultured in TSB (Oxoid) supplemented with $0.25 \%$ glucose at $35^{\circ} \mathrm{C}$ until the turbidity reached McFarland no. 0.5. Approximately $100 \mathrm{cfu}$ of each culture were applied in triplicate into wells of 96-well flat-bottomed microplate containing $200 \mu \mathrm{L}$ of the TSB and $0.25 \%$ glucose. Wells added with cultured S. epidermidis (ATCC12228) served as negative controls. The plate was incubated for $24 \mathrm{~h}$. The content of each well was then discarded and the wells were washed five times with sterile $0.9 \% \mathrm{NaCl}$ solution. Each well surface was stained by adding $100 \mu \mathrm{L}$ of $0.3 \%$ (w/v) crystal violet (Merck) in water and kept for $5 \mathrm{~min}$. After five washing with sterile distilled water and air dried. The biofilm fixed on each well surface was extracted with $100 \mu \mathrm{L}$ of $70 \%$ ethanol and measured the absorbance at $\mathrm{OD}_{570 \mathrm{~nm}}$. The isolates with $\mathrm{OD}_{570 \mathrm{~nm}}$ values above the mean $\mathrm{OD}_{570 \mathrm{~nm}}$ values plus three standard deviations of the negative control $\left(\right.$ mean $\left._{\text {neg }}+3 \mathrm{SD}_{\mathrm{Neg}}\right)$ were considered positive for biofilm formation.

2.8. Statistical Analyses. SPSS Statistics 16.0 was used for statistical analysis. Chi-squared $\left(\chi^{2}\right)$ test and $t$-test were used to analyze the data sorted by MRSA and MSSA groups and frequencies of virulence genes and biofilm formation, respectively. A probability value $(P)<0.05$ was considered different significantly.

\section{Results}

3.1. Antimicrobial Susceptibility. All of the 92 bacterial isolates from culture stocks were verified as $S$. aureus strains according to their phenotypic characteristics determined by the conventional microbiological method. After testing with the $30 \mu \mathrm{g}$ cefoxitin disc, $56 / 92$ isolates $(60.87 \%)$ were MRSA (37 isolates from the Prince of Songkla hospital and 19 isolates from Prasat Neurological Institute), and 36 isolates (39.13\%) were MSSA (5 isolates from the Prince of Songkla hospital, 17 isolates from the Prasat Neurological Institute, and 19 isolates from the Hospital for Tropical Diseases). The 92 S. aureus Thailand isolates were arbitrarily classified into 10 drug groups. Groups 1-7 were MRSA and groups 8-10 were MSSA. Data on susceptible and intermediate sensitivity to the 11 antibiotics tested (cefoxitin, ciprofloxacin, clindamycin, erythromycin, gentamicin, oxacillin, penicillin, rifampin, trimethoprim/sulfamethoxazole (T/S), tetracycline, and teicoplanin) were group 1 (16 isolates): susceptible (9 isolates) and intermediate ( 7 isolates) to rifampin and susceptible to teicoplanin; group 2 (2 isolates): susceptible to gentamicin and teicoplanin, intermediate to rifampin; group 3 (7 isolates): susceptible to gentamicin and teicoplanin; group 4 (1 isolate): susceptible to tetracycline and teicoplanin; group 5 (7 isolates): susceptible to rifampin, trimethoprim/sulfametoxazole, tetracycline, and teicoplanin and susceptible to gentamicin (1 isolate); group 6 (10 isolates): susceptible to rifampin, trimethoprim/sulfametoxazole (10 isolates), intermediate to trimethoprim/sulfametoxazole (1 isolate), and susceptible to teicoplanin; group 7 (13 isolates): susceptible to teicoplanin; group 8 (3 isolates): susceptible to oxacillin ( 2 isolates), cefoxitin, gentamicin, gentamicin, and teicoplanin; group 9 (28 isolates): resistant to penicillin and tetracycline (13 isolates), intermediate to erythromycin (1 islates); group 10 (5 isolates): resistant to gentamicin (1 isolate), ciprofloxacin (2 isolates), erythromycin (2 isolates), and clindamycin ( 2 isolates). All of the isolates were sensitive to vancomycin according to the MIC testing. The methicillin susceptibility and drug groups of the 92 isolates are shown in Table 2.

3.2. Prevalence of Toxin Genes in Individual S. aureus Isolates. Among the 92 isolates, 1 isolate $(1.08 \%)$ did not have any toxin gene (S38), 4 (4.35\%) isolates (S16, S33, S40, and P33) carried one toxin gene (seq), and the remaining 87 isolates (94.57\%) carried two or more toxin genes (Table 2). There were only $6 / 92$ isolates that carry the etx genes either eta or etd (P28, P31, $\mathrm{T} 3, \mathrm{~T} 8, \mathrm{~T} 9$, and $\mathrm{T} 13$ ). The prevalence of toxin genes among the isolates is shown in Figure 1. The predominant enterotoxin gene was seq which was presented in 91/92 isolates (98.91\%), followed by sea (65.22\%) and sek (54.35\%). There was no isolate with see, tsst-1 (sef), or etb. The prevalence of sea, sec, sed, seg, seh, sei, sej, sem, sen, seo, sep, seq, ser, eta, and etd among the MRSA and MSSA isolates were not different. However, the prevalence of seb, sel, and seu among isolates of the two methicillin groups was different significantly.

3.3. Determination of Toxin Production. The bacterial isolates which carried sea, seb, sec, sed; eta and etb; tsst-1 were determined for their ability to produce the respective toxins by using SET-RPLA, TST-RPLA, and EXT-RPLA, respectively, and 35 isolates were toxin producers (Table 2). There were 21/60 sea strains (35\%) that produced SEA; $9 / 13$ seb isolates (69.23\%) produced SEB; 4/7 sec isolates (57.14\%) produced SEC; and $3 / 5$ sed isolates (60\%) produced SED. One of the three eta positive strains $(33.33 \%)$ could produce ETA. None of the four isolates with etd-positive strains produced ETD. Among the MRSA, 24/56 isolates (42.86\%) produced toxins (17 SEA and 7 SEB), whereas 11/36 (30.55\%) of the MSSA isolates produced toxins (SEA 4 isolates, SEB 1 isolate, SEC 3 isolates, SED 2 isolates, and SEB and ETA 1 isolate). There were 3 MSSA isolates that produced more than one toxin: S41 produced SEB and SED, P23 produced SEA and SEC, and T3 produced SEB and ETA.

3.4. PFGE Types. The 92 S. aureus isolates could be classified according to the PFGE results into 23 genotypes, genotypes 1-23 (Figure 2). Information on the PFGE types of individual 
TABle 2: Characteristics of the 92 S. aureus Thailand isolates.

\begin{tabular}{|c|c|c|c|c|c|c|c|c|}
\hline Isolate no. & $\begin{array}{c}\text { Methicillin } \\
\text { susceptibility }\end{array}$ & Drug group & Enterotoxin gene(s) & ExT gene & RPLA toxin & PFGE type & Agr group & Biofilm (OD) \\
\hline S1 & $\mathrm{R}$ & 1 & sek, seq & - & ND & 1 & 1 & $+(0.831)$ \\
\hline S2 & $\mathrm{R}$ & 1 & sea, sek, seo, seq & - & $\mathrm{ND}$ & 1 & 1 & $+(0.828)$ \\
\hline S3 & $\mathrm{R}$ & 1 & sek, seq & - & ND & 1 & 1 & $+(0.039)$ \\
\hline S4 & $\mathrm{R}$ & 1 & sek, seq & - & ND & 1 & 1 & $+(0.181)$ \\
\hline S5 & $\mathrm{R}$ & 1 & sea, sek, seq & - & - & 1 & 1 & $+(0.701)$ \\
\hline S6 & $\mathrm{R}$ & 1 & sek, seq & - & $\mathrm{ND}$ & 2 & 1 & $+(1.566)$ \\
\hline S7 & $\mathrm{R}$ & 1 & sea, sed, sek, seq & - & - & 1 & 2 & $+(1.841)$ \\
\hline S8 & $\mathrm{R}$ & 1 & sea, sek, seq & - & SEA & 6 & 1 & $+(1.701)$ \\
\hline S9 & $\mathrm{R}$ & 1 & sea, sek, seq & - & SEA & 21 & 1 & $+(0.996)$ \\
\hline S10 & $\mathrm{R}$ & 1 & sea, sek, seq & - & SEA & 21 & 1 & $+(1.219)$ \\
\hline S11 & $\mathrm{R}$ & 1 & sea, sek, seo, seq & - & SEA & 21 & 1 & $+(1.749)$ \\
\hline S12 & $\mathrm{R}$ & 1 & sea, sek, seq & - & SEA & 21 & 1 & $+(1.377)$ \\
\hline S13 & $\mathrm{R}$ & 1 & sea, sek, seq & - & SEA & 21 & 1 & $+(1.687)$ \\
\hline S14 & $\mathrm{R}$ & 1 & sea, sek, seq & - & SEA & 21 & 1 & $+(0.796)$ \\
\hline S15 & $\mathrm{R}$ & 2 & sea, sek, seq & - & - & 1 & 1 & $+(0.097)$ \\
\hline S16 & $\mathrm{R}$ & 2 & seq & - & ND & 3 & 1 & $+(0.132)$ \\
\hline S17 & $\mathrm{R}$ & 3 & sea, sek, seq & - & - & 1 & 1 & $+(0.085)$ \\
\hline S18 & $\mathrm{R}$ & 3 & sea, sek, seq & - & - & 4 & 1 & $+(0.230)$ \\
\hline S19 & $\mathrm{R}$ & 3 & sea, sek, seq & - & - & 4 & 1 & $+(0.080)$ \\
\hline S20 & $\mathrm{R}$ & 3 & sek, seq & - & $\mathrm{ND}$ & 6 & 1 & $+(0.417)$ \\
\hline S21 & $\mathrm{R}$ & 3 & sek, seq & - & ND & 6 & 1 & $+(1.103)$ \\
\hline S22 & $\mathrm{R}$ & 3 & sek, seq & - & ND & 6 & 1 & $+(1.835)$ \\
\hline S23 & $\mathrm{R}$ & 3 & sek, seq & - & $\mathrm{ND}$ & 21 & 1 & $+(0.097)$ \\
\hline S24 & $\mathrm{R}$ & 4 & sea, sek, seq & - & - & 2 & 1 & $+(0.552)$ \\
\hline S25 & $\mathrm{R}$ & 7 & sea, sek, seq & - & - & 1 & 1 & $+(0.569)$ \\
\hline S26 & $\mathrm{R}$ & 7 & sek, seq & - & $\mathrm{ND}$ & 1 & 1 & $+(1.000)$ \\
\hline S27 & $\mathrm{R}$ & 7 & sek, seq & - & ND & 1 & 1 & $+(1.155)$ \\
\hline S28 & $\mathrm{R}$ & 7 & sea, sek, seq & - & - & 1 & 1 & $+(0.715)$ \\
\hline S29 & $\mathrm{R}$ & 7 & sek, seq & - & $\mathrm{ND}$ & 2 & 1 & $+(1.061)$ \\
\hline S30 & $\mathrm{R}$ & 7 & sek, seq & - & ND & 2 & 1 & $+(1.131)$ \\
\hline S31 & $\mathrm{R}$ & 7 & sek, seq & - & $\mathrm{ND}$ & 2 & 1 & $+(0.774)$ \\
\hline S32 & $\mathrm{R}$ & 7 & sea, sec, sek, sel, seq & - & - & 9 & 1 & $+(1.796)$ \\
\hline S33 & $\mathrm{R}$ & 7 & seq & - & ND & 21 & 1 & $+(2.481)$ \\
\hline S34 & $\mathrm{R}$ & 7 & sea, sec, sel, seq & - & - & 21 & 1 & $+(1.000)$ \\
\hline S35 & $\mathrm{R}$ & 7 & sea, sek, seq & - & - & 21 & 1 & $+(1.792)$ \\
\hline S36 & $\mathrm{R}$ & 7 & sek, seq & - & $\mathrm{ND}$ & 21 & 1 & $+(1.184)$ \\
\hline S37 & $\mathrm{R}$ & 7 & sek, seq & - & ND & 21 & 1 & $+(2.332)$ \\
\hline S38 & S & 8 & & - & $\mathrm{ND}$ & 4 & 1 & $-(-0.052)$ \\
\hline S39 & S & 8 & sej, sek, seq & - & ND & 4 & 2 & $+(0.367)$ \\
\hline S40 & S & 8 & seq & - & ND & 21 & 1 & $+(0.508)$ \\
\hline S41 & S & 9 & seb, sed, sej, sek, seq, ser, etd & - & SEB, SED & 21 & 3 & $+(0.074)$ \\
\hline S42 & S & 9 & seg, sei, sem, sen, seo, seq, seu & - & - & 19 & 3 & $-(-0.007)$ \\
\hline $\mathrm{S} 43$ & $\mathrm{~S}$ & 9 & seg, sei, sek, sem, sen, seo, seq & - & - & 7 & 2 & $-(-0.008)$ \\
\hline $\mathrm{P} 1$ & $\mathrm{R}$ & 1 & sea, seq & - & SEA & 21 & 1 & $+(0.317)$ \\
\hline $\mathrm{P} 2$ & $\mathrm{R}$ & 1 & sea, seg, sei, sek, sen, seo, seq & - & SEA & 9 & 2 & $+(0.700)$ \\
\hline
\end{tabular}


TABLe 2: Continued.

\begin{tabular}{|c|c|c|c|c|c|c|c|c|}
\hline Isolate no. & $\begin{array}{c}\text { Methicillin } \\
\text { susceptibility }\end{array}$ & Drug group & Enterotoxin gene(s) & ExT gene & RPLA toxin & PFGE type & Agr group & Biofilm (OD) \\
\hline P3 & $\mathrm{R}$ & 5 & sea, seg, sei, sem, sen, seo, seq & - & SEA & 9 & 2 & $+(0.098)$ \\
\hline $\mathrm{P} 4$ & $\mathrm{R}$ & 5 & sea, seg, sei, sem, sen, seo, seq & - & SEA & 9 & 2 & $-(-0.194)$ \\
\hline P5 & $\mathrm{R}$ & 5 & sea, sei, sek, sen, seo, seq & - & SEA & 9 & 2 & $+(0.543)$ \\
\hline P6 & $\mathrm{R}$ & 5 & sea, seg, sei, sem, sen, seo, seq & - & SEA & 9 & 2 & $-(-0.144)$ \\
\hline P7 & $\mathrm{R}$ & 5 & $\begin{array}{c}\text { sea, seg, sei, sek, sem, sen, seo, } \\
\text { seq }\end{array}$ & - & SEA & 9 & 2 & $-(-0.095)$ \\
\hline P8 & $\mathrm{R}$ & 5 & sea, seg, sei, sem, sen, seo, seq & - & SEA & 13 & 2 & $+(0.451)$ \\
\hline P9 & $\mathrm{R}$ & 5 & $\begin{array}{c}\text { sea, sed, seg, sei, sej, sem, sen, } \\
\text { seo, sep, seq, ser }\end{array}$ & - & SED & 16 & 2 & $-(-0.05)$ \\
\hline $\mathrm{P} 10$ & $\mathrm{R}$ & 6 & sea, sek, seq & - & SEA & 21 & 1 & $+(0.817)$ \\
\hline P11 & $\mathrm{R}$ & 6 & $\begin{array}{c}\text { sea, seb, seg, sei, sem, sen, seo, } \\
\text { seq }\end{array}$ & - & SEB & 22 & 2 & $+(0.141)$ \\
\hline $\mathrm{P} 12$ & $\mathrm{R}$ & 6 & seb, seg, sei, sem, sen, seo, seq & - & SEB & 22 & 2 & $+(0.179)$ \\
\hline $\mathrm{P} 13$ & $\mathrm{R}$ & 6 & seb, seg, sei, sem, sen, seq & - & SEB & 22 & 2 & $-(-0.176)$ \\
\hline P14 & $\mathrm{R}$ & 6 & $\begin{array}{c}\text { sea, seb, seg, sei, sem, sen, seo, } \\
\text { seq }\end{array}$ & - & - & 22 & 2 & $+(0.182)$ \\
\hline P15 & $\mathrm{R}$ & 6 & $\begin{array}{c}\text { sea, seb, seg, sei, sem, sen, seo, } \\
\text { seq }\end{array}$ & - & SEB & 22 & 2 & $-(-0.084)$ \\
\hline P16 & $\mathrm{R}$ & 6 & $\begin{array}{c}\text { sea, seb, seg, sei, sem, sen, seo, } \\
\text { seq }\end{array}$ & - & SEB & 22 & 2 & $-(-0.249)$ \\
\hline P17 & $\mathrm{R}$ & 6 & seb, seg, sei, sem, sen, seo, seq & - & SEB & 22 & 2 & $-(-0.051)$ \\
\hline $\mathrm{P} 18$ & $\mathrm{R}$ & 6 & seb, seg, sei, sem, sen, seo, seq & - & SEB & 22 & 2 & $-(-0.137)$ \\
\hline P19 & $\mathrm{R}$ & 6 & $\begin{array}{c}\text { sea, seb, seg, sei, sem, sen, seo, } \\
\text { seq }\end{array}$ & - & - & 22 & 2 & $-(-0.117)$ \\
\hline P20 & S & 9 & sea, sek, sel, seq & - & - & 1 & 1 & $+(1.311)$ \\
\hline $\mathrm{P} 21$ & $\mathrm{~S}$ & 9 & sea, sec, sel, seq & - & - & 2 & 1 & $-(-0.173)$ \\
\hline $\mathrm{P} 22$ & S & 9 & sea, seo, seq & - & - & 8 & 1 & $+(0.300)$ \\
\hline P23 & S & 9 & sea, sec, sel, seq & - & SEA, SEC & 9 & 1 & $-(-0.204)$ \\
\hline $\mathrm{P} 24$ & S & 9 & sea, seq & - & - & 9 & 1 & $+(2.210)$ \\
\hline P25 & S & 9 & sea, sek, seq & - & - & 10 & 4 & $+(0.484)$ \\
\hline P26 & $\mathrm{S}$ & 9 & sea, sek, seo, seq & - & - & 17 & 1 & $+(1.156)$ \\
\hline P27 & S & 9 & sea, seh, sek, seq & - & SEA & 17 & 3 & $-(-0.058)$ \\
\hline P28 & $\mathrm{S}$ & 9 & sea, sed, sei, seq & etd & SED & 18 & 1 & $-(-0.225)$ \\
\hline P29 & S & 9 & sea, seq & - & - & 18 & 2 & $+(0.098)$ \\
\hline P30 & $\mathrm{S}$ & 9 & $\begin{array}{c}\text { sea, sec, seg, sei, sel, sem, sen, } \\
\text { seo, seq }\end{array}$ & - & SEC & 18 & 2 & $-(-0.390)$ \\
\hline P31 & $S$ & 9 & $\begin{array}{c}\text { sea, seb, seg, sei, sem, sen, seo, } \\
\text { seq, seu }\end{array}$ & eta & - & 18 & 2 & $-(-0.147)$ \\
\hline $\mathrm{P} 32$ & S & 9 & sea, sec, seg, sei, sel, sen, seo, seq & - & SEC & 20 & 3 & $+(0.128)$ \\
\hline P33 & S & 9 & seq & - & ND & 20 & 3 & $-(-0.245)$ \\
\hline P34 & S & 9 & seo, seq & - & ND & 22 & 1 & $-(-0.326)$ \\
\hline P35 & S & 9 & sea, seo, seq & - & - & 23 & 1 & $+(0.054)$ \\
\hline P36 & $\mathrm{S}$ & 9 & sed, sek, seq & - & ND & 23 & 1 & $+(1.107)$ \\
\hline $\mathrm{T} 1$ & S & 9 & sea, sen, seq & - & - & 5 & 2 & $-(-0.073)$ \\
\hline $\mathrm{T} 2$ & S & 9 & sea, seg, sem, sen, seo, seq & - & SEA & 5 & 2 & $+(0.046)$ \\
\hline $\mathrm{T} 3$ & $S$ & 9 & $\begin{array}{c}\text { seb, seg, sei, sem, sen, seo, seq, } \\
\text { seu }\end{array}$ & eta & SEB, ETA & 8 & 4 & $+(3.872)$ \\
\hline
\end{tabular}


TABLE 2: Continued.

\begin{tabular}{|c|c|c|c|c|c|c|c|c|}
\hline Isolate no. & $\begin{array}{c}\text { Methicillin } \\
\text { susceptibility }\end{array}$ & Drug group & Enterotoxin gene(s) & ExT gene & RPLA toxin & PFGE type & Agr group & Biofilm (OD) \\
\hline $\mathrm{T} 4$ & S & 9 & sea, seg, sen, seq & - & - & 9 & 2 & $+(0.319)$ \\
\hline T5 & S & 9 & $\begin{array}{c}\text { sea, seg, sei, sek, sem, sen, seo, } \\
\text { seq, seu }\end{array}$ & - & - & 9 & 4 & $-(-0.081)$ \\
\hline T6 & S & 9 & seg, sen, seq & - & ND & 11 & 1 & $+(0.736)$ \\
\hline $\mathrm{T} 7$ & S & 9 & sea, seg, sei, sek, sen, seo & - & - & 15 & 2 & $+(2.818)$ \\
\hline $\mathrm{T} 8$ & S & 9 & sea, seg, sei, sem, sen, seo, seq & eta & - & 18 & 1 & $+(0.156)$ \\
\hline T9 & S & 10 & seq & etd & ND & 12 & 1 & $-(-0.114)$ \\
\hline $\mathrm{T} 10$ & S & 10 & sea, seg, sei, sem, sen, seo, seq & - & SEA & 14 & 2 & $+(0.086)$ \\
\hline T11 & S & 10 & sea, seg, sei, sem, sen, seo, seq & - & - & 15 & 2 & $+(0.808)$ \\
\hline $\mathrm{T} 12$ & S & 10 & sec, seh, sel, seq & - & SEC & 15 & 3 & $-(-0.198)$ \\
\hline $\mathrm{T} 13$ & S & 10 & sea, sek, seq & etd & SEA & 18 & 1 & $+(0.235)$ \\
\hline
\end{tabular}

-: not detectable, +: produced biofilm.

ND: not done.

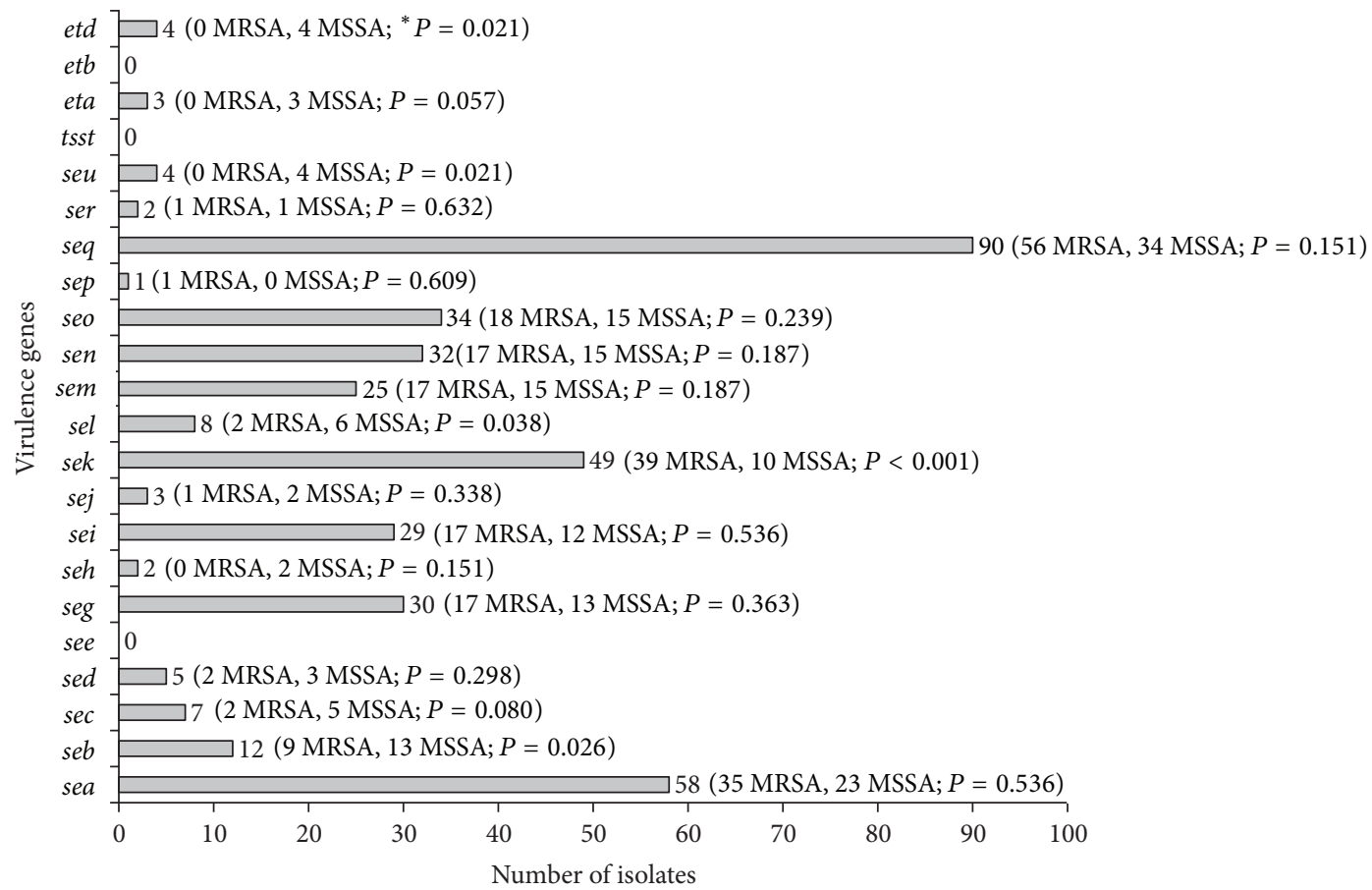

Figure 1: Prevalence of the enterotoxin and exfoliative toxin genes among the 92 S. aureus Thailand isolates. ${ }^{*} P$ value between prevalence of MRSA compared to MSSA.

isolates is given in Table 2. PFGE type 21 was predominant (16 isolates), followed by types 1,9 , and $22(13,11$, and 10 isolates, resp.); types 2 and 18 had 6 isolates each; types 4 and 6 had 4 isolates each; 3 isolates belonged to type 15; types $5,8,17,20$, and 23 had 2 isolates each, and types $3,7,10,11,12,13,14,16$, and 19 had 1 isolate each.

3.5. The Agr Groups. The predominant agr group among the 92 isolates was group 1 (54/92 isolates; $58.70 \%)$ followed by groups 2 (29 isolates; $31.52 \%$ ), 3 (6 isolates; $6.52 \%$ ), and 4 (3 isolates; $3.26 \%$ ).
3.6. Biofilm Formation. There were $67 / 92$ isolates (72.83\%) that produced biofilm; $21 / 36$ (58.33\%) were MSSA and 46/56 isolates $(82.14 \%)$ were MRSA. The prevalence of the biofilm formation of the MRSA and MSSA was not different $(P>$ 0.05).

\section{Discussion}

Diseases caused by $S$. aureus are health hazard to human worldwide. Since the first recognition of methicillin-resistant S. aureus in 1961 [29], there has been an upsurge of infections 

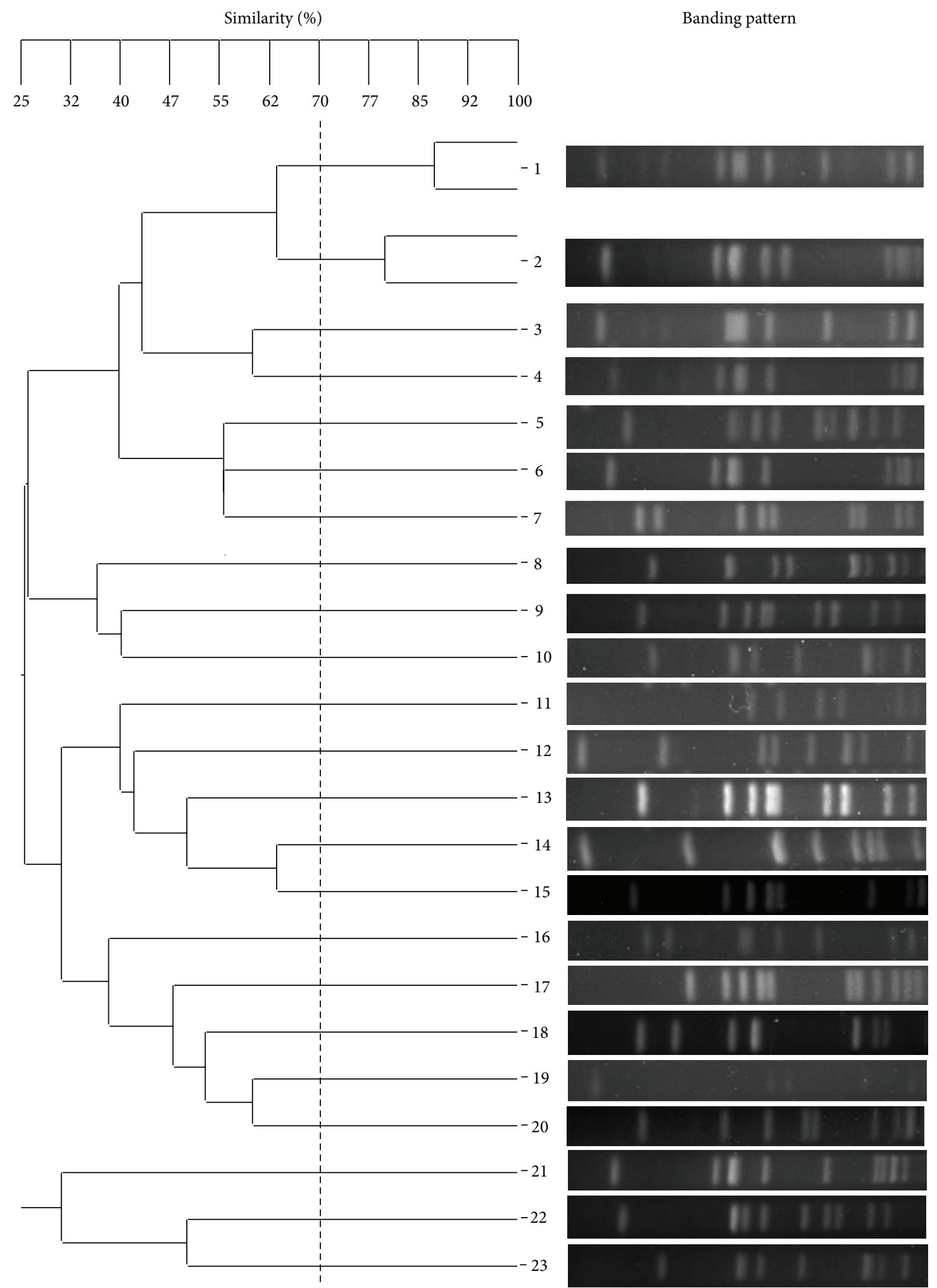

FIGURE 2: Dendrogram of PFGE patterns the 92 S. aureus Thailand isolates.

caused by the $S$. aureus variants that resist not only methicillin, but also other $\beta$-lactams and vancomycin, which are therapeutic drugs of choice [30-32], leading to treatment failure and increased case fatality rate. The methicillin and vancomycin resistance of the $S$. aureus are encoded by staphylococcal cassette chromosome mec (SCCmec) and vanA, respectively $[30,31]$. Association of the presence of $S$. aureus toxin genes with methicillin sensitivity and resistance 
among S. aureus has been reported previously [28, 33-35]. The association was found also in the present study; the prevalence of the $s e b, s e c, s e l, s e u$, and eta was associated significantly $(P<0.05)$ with the MSSA while sek was found more in MRSA.

The toxin genes carried by the 92 Thailand isolates varied from none to as many as 11 genes (Table 2). Five of the $S$. aureus enterotoxin genes, that is, seg, sei, sem, sen, and seo, belonged to the highly prevalent egc locus [36, 37]; thus, their coexistence was frequently reported. Coexistence of segsei in the same strain, either alone or in more combination with other toxin gene(s) (sea, sec, sed, seh, sej, and/or tst) was found in 55\% of the 429 S. aureus isolates from Germany [38]. In Japan, the seg-sei alone or with seb, sec, or sed were 24, 2.7, 6.8, and 2.0\%, respectively [39]. The combined seg-seisem-sen-seo with seu was $15.1 \%$ among the Chinese isolates [26]. In the present study, the combined seg-sei-sem-sen-seo with other toxin genes including sea, seb, sed, sej, sek, sel, sep, seq, ser, and/or eta was found in 24/92 isolates (26.1\%). There were 3 isolates that carried seg-sei-sen-seo with sea, sec, sek, sel, and/or seq and 1 isolate with seg-sei-sem-sen and seb. The previously reported fixed association of sed-sej [38] was not found among the 92 Thailand isolates. The combined sek-seq with other toxin gene(s), that is, sea and/or seb, was $45.5 \%$ among the Chinese isolates [26]. In the present study, the sekseq association was found in 48 of the 92 isolates $(52.17 \%)$, either the two genes alone $(16.3 \%)$ or with the other toxin genes $(35.86 \%)$.

The ability of the isolates to produce SEA, SEB, SEC, and SED and ETA, ETB, and TSST-1 was examined by using SET-RPLA, TST-RPLA, and EXT-RPLA test kits, respectively. Not all isolates harboring the genes expressed the respective toxins. The results were similar to the finding reported previously among $S$. aureus isolates from milk and milk products from Morocco [40]. The unconformed results between genotypes (by PCR) to phenotypes (by RPLA) could be due to the fact that toxin production of the bacteria can be affected by the growth conditions including temperature, $\mathrm{pH}$, and water activity. The so-produced toxin levels might be lower than the detection limits of the immunoassay [40, 41]. Alternatively, the toxin gene may not be expressed due to mutation either in the coding region or in a regulatory region, for example, agr [42, 43]. No annotated data are available in the literature on association of the ability of toxin production and antibiograms of the $S$. aureus. Nevertheless, in this study, the frequency of toxin production is higher among the MRSA (48.86\%) than the MSSA (30.55\%) $(P<$ $0.05)$.

There was no association between PFGE patterns with the MRSA and MSSA of the 92 Thai strains which conformed to the results reported elsewhere [44, 45]. However, PFGE patterns 21 and 22 of MRSA strains predominated among isolates from Prince of Songkla Hospital and Prasat Neurological Institute, that is, 32.5 and $27.8 \%$, respectively. Among the 7 isolates of PFGE pattern 21 of Songkla that could produce enterotoxins, 6 strains (85.7\%) produced SEA. All 7 isolates of PFGE type 22 of Prasat Neurological Institute isolates produced SEB.

The polymorphism in the agr locus was first described by Ji et al. in 1997 [46]. To date, S. aureus isolates were classified into four different agr groups $[25,46]$. In this study, all $a g r$ groups were found; large proportion (58.6\%) of the isolates was agr group 1 which was similar to the data reported previously [16]. Moreover, majority (38/54 isolates, 70\%) of the agr group 1 were MRSA which conformed also to the previous report [47]. However, it is noteworthy that isolates of the agr group 2 in this study carried more number of enterotoxins genes, and most of the toxin producing strains belonged to this agr group. The data were different from elsewhere which showed that most toxin producing $S$. aureus strains were either agr groups 3 [46] or 4 [48].

Biofilm formation contributes to bacterial pathogenesis and resistance to antibiotics and harsh environment. S. aureus isolates did form biofilms [28, 49]. More strains of MSSA produced biofilm compared to MRSA strains [28]. In this study, $72.83 \%$ of the $S$. aureus isolates formed biofilm but there was no association with their antibiotic patterns.

In conclusion, the results of this study provide insight information on molecular and phenotypic markers of $S$. aureus clinical isolates in Thailand which should be useful for future active surveillance that aimed to control a spread of existing antimicrobial resistant bacteria as well as early recognition of a newly emerged variant.

\section{Conflict of Interests}

The authors have declared that no conflict of interests exists.

\section{Acknowledgments}

The work was supported by DPG5380001 grant, the Thailand Research Fund (TRF), and the National Research University (NRU) Project of the Office of Higher Education Commission, Ministry of Education, Thailand. Nitaya Indrawattana and Nitat Sookrung are the TRF new scholars. Thanks are due to Assistant Professor Narisara Chantratita, Department of Microbiology and Immunology, Faculty of Tropical Medicine, Mahidol University, Bangkok, for providing the PFGE facility.

\section{References}

[1] R. E. Williams, "Healthy carriage of Staphylococcus aureus: its prevalence and importance," Bacteriological reviews, vol. 27, pp. 56-71, 1963.

[2] D. L. Stulberg, M. A. Penrod, and R. A. Blatny, "Common bacterial skin infections," The American Family Physician, vol. 66, no. 1, pp. 119-124, 2002.

[3] I. V. Pinchuk, E. J. Beswick, and V. E. Reyes, "Staphylococcal enterotoxins," Toxins, vol. 2, no. 8, pp. 2177-2197, 2010.

[4] S. D. Haessler and R. B. Brown, "Pneumonia caused by Staphylococcus aureus," Current Respiratory Medicine Reviews, vol. 5, no. 1, pp. 62-67, 2009. 
[5] M. Kowalik, J. Ellert-Zygadłowska, T. Smiatacz et al., "Staphylococcus aureus sepsis: still life threatening disease," Polski Merkuriusz Lekarski, vol. 11, no. 64, pp. 352-356, 2001.

[6] T. Proft and J. D. Fraser, "Bacterial superantigens," Clinical and Experimental Immunology, vol. 133, no. 3, pp. 299-306, 2003.

[7] M. M. Dinges, P. M. Orwin, and P. M. Schlievert, "Exotoxins of Staphylococcus aureus," Clinical Microbiology Reviews, vol. 13, no. 1, pp. 16-34, 2000.

[8] N. Balaban and A. Rasooly, "Staphylococcal enterotoxins," International Journal of Food Microbiology, vol. 61, no. 1, pp. 1$10,2000$.

[9] M. Llewelyn and J. Cohen, "Superantigens: microbial agents that corrupt immunity," The Lancet Infectious Diseases, vol. 2, no. 3, pp. 156-162, 2002

[10] G. Lina, G. A. Bohach, S. P. Nair, K. Hiramatsu, E. JouvinMarche, and R. Mariuzza, "Standard nomenclature for the superantigens expressed by Staphylococcus," Journal of Infectious Diseases, vol. 189, no. 12, pp. 2334-2336, 2004.

[11] M. Á. Argudín, M. C. Mendoza, and M. R. Rodicio, "Food poisoning and Staphylococcus aureus enterotoxins," Toxins, vol. 2, no. 7, pp. 1751-1773, 2010.

[12] M. S. Bergdoll, B. A. Crass, R. F. Reiser, R. N. Robbins, and J. P. Davis, "A new staphylococcal enterotoxin, enterotoxin F, associated with toxic-shock-syndrome Staphylococcus aureus isolates," The Lancet, vol. 1, no. 8228, pp. 1017-1021, 1981.

[13] S. Holtfreter, D. Grumann, M. Schmudde et al., "Clonal distribution of superantigen genes in clinical Staphylococcus aureus isolates," Journal of Clinical Microbiology, vol. 45, no. 8, pp. 2669-2680, 2007.

[14] R. P. Novick, H. F. Ross, S. J. Projan, J. Kornblum, B. Kreiswirth, and S. Moghazeh, "Synthesis of staphylococcal virulence factors is controlled by a regulatory RNA molecule," EMBO Journal, vol. 12, no. 10, pp. 3967-3975, 1993.

[15] N. Balaban and R. P. Novick, "Translation of RNAIII, the Staphylococcus aureus, agr regulatory RNA molecule, can be activated by a $3^{\prime}$-end deletion," FEMS Microbiology Letters, vol. 133, no. 1-2, pp. 155-161, 1995.

[16] P. Gilot, G. Lina, T. Cochard, and B. Poutrel, "Analysis of the genetic variability of genes encoding the RNA III-activating components Agr and TRAP in a population of Staphylococcus aureus strains isolated from cows with mastitis," Journal of Clinical Microbiology, vol. 40, no. 11, pp. 4060-4067, 2002.

[17] F. D. Lowy, "Medical progress: Staphylococcus aureus infections," The New England Journal of Medicine, vol. 339, no. 8, pp. 520-532, 1998.

[18] F. D. Lowy, "Antimicrobial resistance: the example of Staphylococcus aureus," Journal of Clinical Investigation, vol. 111, no. 9, pp. 1265-1273, 2003.

[19] Y. Katayama, T. Ito, and K. Hiramatsu, "A new class of genetic element, staphylococcus cassette chromosome mec, encodes methicillin resistance in Staphylococcus aureus," Antimicrobial Agents and Chemotherapy, vol. 44, no. 6, pp. 1549-1555, 2000.

[20] The committee on Using Computer in Clinical Microbiology, "Bacterial antimicrobial susceptibility pattern 1998," Journal of Infectious Diseases and Antimicrobial Agents, vol. 8, pp. 25-39, 1991.

[21] “Drug resistance surveillance 1998, pilot phase report," National Antimicrobial Resistance Surveillance Center, Ministry of Public Health, Thailand, 1999.

[22] S. Wongwanich, P. Tishyadhigama, S. Paisomboon, T. Ohta, and H. Hayashi, "Epidemiological analysis of methicillin resistant
Staphylococcus aureus in Thailand," Southeast Asian Journal of Tropical Medicine and Public Health, vol. 31, no. 1, pp. 72-76, 2000.

[23] S. Trakulsomboon, S. Danchaivijitr, Y. Rongrungruang et al., "First report of methicillin-resistant Staphylococcus aureus with reduced susceptibility to vancomycin in Thailand," Journal of Clinical Microbiology, vol. 39, no. 2, pp. 591-595, 2001.

[24] CLSI, "Performance standards for antimicrobial susceptibility testing, 20th informational supplement," CLSI Document M100-S20, CLSI, Wayne, Pa, USA, 2010.

[25] S. Jarraud, G. J. Lyon, A. M. S. Figueiredo et al., "Exfoliatinproducing strains define a fourth agr specificity group in Staphylococcus aureus," Journal of Bacteriology, vol. 182, no. 22, pp. 6517-6522, 2000.

[26] D. Wu, X. Li, Y. Yang et al., "Superantigen gene profiles and presence of exfoliative toxin genes in community-acquired meticillin-resistant Staphylococcus aureus isolated from Chinese children," Journal of Medical Microbiology, vol. 60, no. 1, pp. 35-45, 2011.

[27] S. J. Peacock, G. D. I. de Silva, A. Justice et al., "Comparison of multilocus sequence typing and pulsed-field gel electrophoresis as tools for typing Staphylococcus aureus isolates in a microepidemiological setting," Journal of Clinical Microbiology, vol. 40, no. 10, pp. 3764-3770, 2002.

[28] L. Wang, F. Yu, L. Yang et al., "Prevalence of virulence genes and biofilm formation among Staphylococcus aureus clinical isolates associated with lower respiratory infection," African Journal of Microbiology Research, vol. 4, no. 23, pp. 2566-2569, 2010.

[29] M. P. Jevons, “Celbenin-resistant staphylococci," British Medical Journal, vol. 1, pp. 24-25, 1961.

[30] J. B. Kaper and J. Hacker, Pathogenicity Islands and Other Mobile Virulence Elements, The American Society for Microbiology Press, Washington, DC, USA, 1999.

[31] S. Chang, D. M. Sievert, J. C. Hageman et al., "Infection with vancomycin-resistant Staphylococcus aureus containing the vanA resistance gene," The New England Journal of Medicine, vol. 348, no. 14, pp. 1342-1347, 2003.

[32] S. Christopher, R. M. Verghis, B. Antonisamy et al., “Transmission dynamics of methicillin-resistant Staphylococcus aureus in a medical intensive care unit in India," PLoS ONE, vol. 6, no. 7, Article ID e20604, 2011.

[33] J. Sila, P. Sauer, and M. Kolar, "Comparison of the prevalence of genes coding for enterotoxins, exfoliatins, PantonValentine leukocidin and TSST-1 between methicillin-resistant and methicillin-susceptible isolates of Staphylococcus aureus at the University Hospital in Olomouc," Biomedical Papers, vol. 153, no. 3, pp. 215-218, 2009.

[34] K. T. Lim, Y. A. Hanifah, M. Y. Mohd Yusof, and K. L. Thong, "Investigation of toxin genes among methicillinresistant Staphylococcus aureus strains isolated from a tertiary hospital in Malaysia," Tropical Biomedicine, vol. 29, pp. 212-219, 2012.

[35] J. N. Jiménez, A. M. Ocampo, J. M. Vanegas et al., "Characterisation of virulence genes in methicillin susceptible and resistant Staphylococcus aureus isolates from a paediatric population in a university hospital of Medellín, Colombia," Memórias do Instituto Oswaldo Cruz, vol. 106, pp. 980-985, 2011.

[36] S. Jarraud, M. A. Peyrat, A. Lim et al., "egc, a highly prevalent operon of enterotoxin gene, forms a putative nursery of superantigens in Staphylococcus aureus," Journal of Immunology, vol. 166, no. 1, pp. 669-677, 2001. 
[37] M. Mempel, G. Lina, M. Hojka et al., "High prevalence of superantigens associated with the egc locus in Staphylococcus aureus isolates from patients with atopic eczema," European Journal of Clinical Microbiology and Infectious Diseases, vol. 22, no. 5, pp. 306-309, 2003.

[38] K. Becker, A. W. Friedrich, G. Lubritz, M. Weilert, G. Peters, and C. von Eiff, "Prevalence of genes encoding pyrogenic toxin superantigens and exfoliative toxins among strains of Staphylococcus aureus isolated from blood and nasal specimens," Journal of Clinical Microbiology, vol. 41, no. 4, pp. 1434-1439, 2003.

[39] K. Omoe, D.-L. Hu, H. Takahashi-Omoe, A. Nakane, and K. Shinagawa, "Comprehensive analysis of classical and newly described staphylococcal superantigenic toxin genes in Staphylococcus aureus isolates," FEMS Microbiology Letters, vol. 246, no. 2, pp. 191-198, 2005.

[40] A. Bendahou, M. Abid, N. Bouteldoun, D. Catelejine, and M. Lebbadi, "Enterotoxigenic coagulase positive Staphylococcus in milk and milk products, Lben and Jben, in northern Morocco," Journal of Infection in Developing Countries, vol. 3, no. 3, pp. 169176, 2009.

[41] C. A. Genigeorgis, "Present state of knowledge on staphylococcal intoxication," International Journal of Food Microbiology, vol. 9, no. 4, pp. 327-360, 1989.

[42] L. Chapaval, D. H. Moon, J. E. Gomes, F. R. Duarte, and S. M. Tsai, "Use of PCR to detect classical enterotoxin genes (ent) and toxic shock syndrome toxin-1 gene (tst) in Staphylocooccus aureus isolated from crude milk and determination of toxin productivities of $S$. aureus isolates harboring these gene," Arquivos Do Instituto Biológico, vol. 73, pp. 165-169, 2006.

[43] N. K. Sharma, C. E. D. Rees, and C. E. R. Dodd, "Development of a single-reaction multiplex PCR toxin typing assay for Staphylococcus aureus strains," Applied and Environmental Microbiology, vol. 66, no. 4, pp. 1347-1353, 2000.

[44] Y. Xie, Y. He, A. Gehring et al., "Genotypes and toxin gene profiles of Staphylococcus aureus clinical isolates from China," PLoS ONE, vol. 6, no. 12, Article ID e28276, 2011.

[45] Y. Shimamura and M. Murata, "Pulsed-field gel electrophoretic analysis and some characteristics of Staphylococcus aureus isolated from retail foods and human hands," Bioscience, Biotechnology and Biochemistry, vol. 75, no. 6, pp. 1177-1180, 2011.

[46] G. Ji, R. Beavis, and R. P. Novick, "Bacterial interference caused by autoinducing peptide variants," Science, vol. 276, no. 5321, pp. 2027-2030, 1997.

[47] W. van Leeuwen, W. van Nieuwenhuizen, C. Gijzen, H. Verbrugh, and A. van Belkum, "Population studies of methicillinresistant and -sensitive Staphylococcus aureus strains reveal a lack of variability in the agrD gene, encoding a staphylococcal autoinducer peptide," Journal of Bacteriology, vol. 182, no. 20, pp. 5721-5729, 2000.

[48] S. Jarraud, C. Mougel, J. Thioulouse et al., "Relationships between Staphylococcus aureus genetic background, virulence factors, agr groups (alleles), and human disease," Infection and Immunity, vol. 70, no. 2, pp. 631-641, 2002.

[49] G. F. M. Gad, M. A. El-Feky, M. S. El-Rehewy, M. A. Hassan, H. Abolella, and R. M. A. El-Baky, "Detection of icaA, icaD genes and biofilm production by Staphylococcus aureus and Staphylococcus epidermidis isolated from urinary tract catheterized patients," Journal of Infection in Developing Countries, vol. 3, no. 5, pp. 342-351, 2009. 

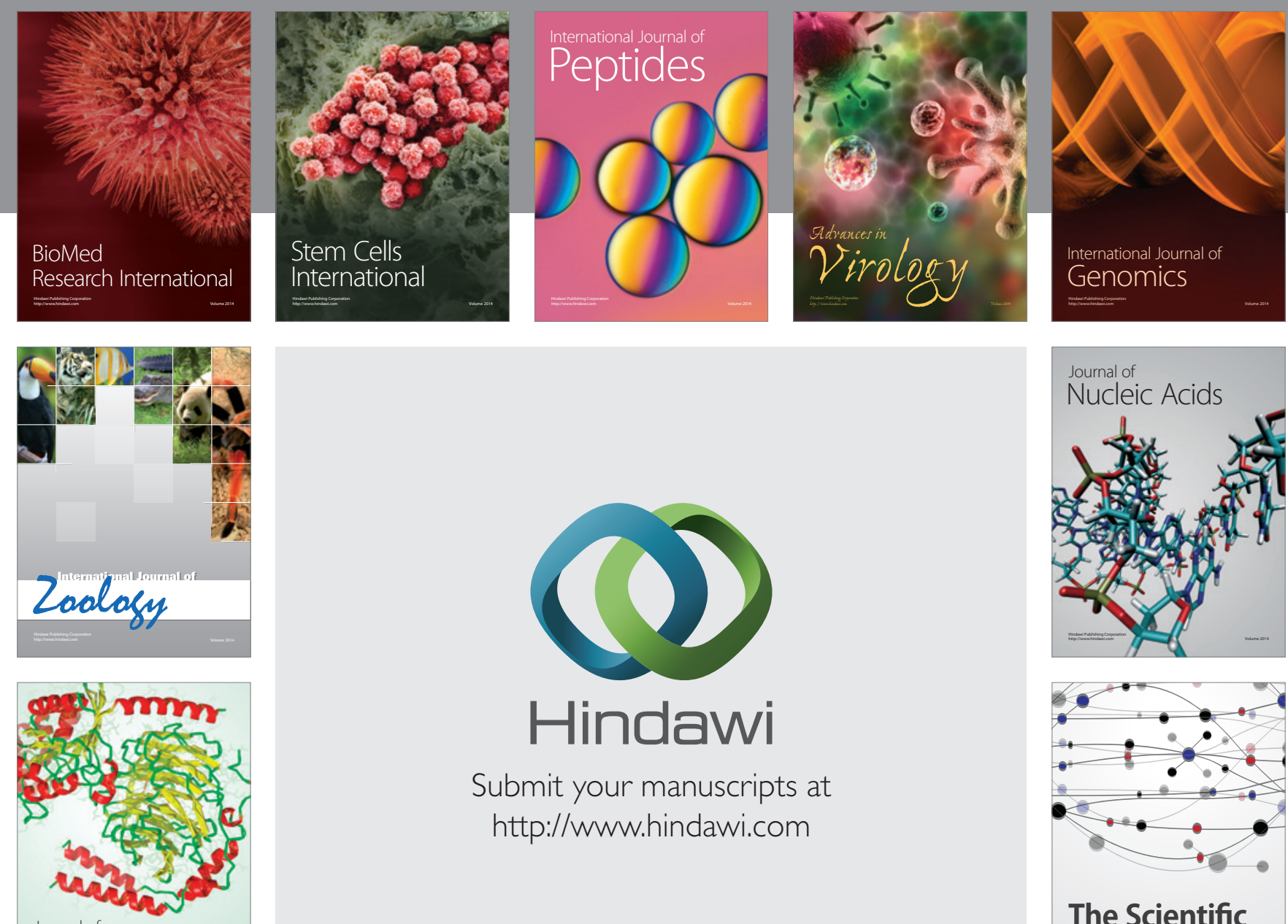

Submit your manuscripts at

http://www.hindawi.com

Journal of
Signal Transduction
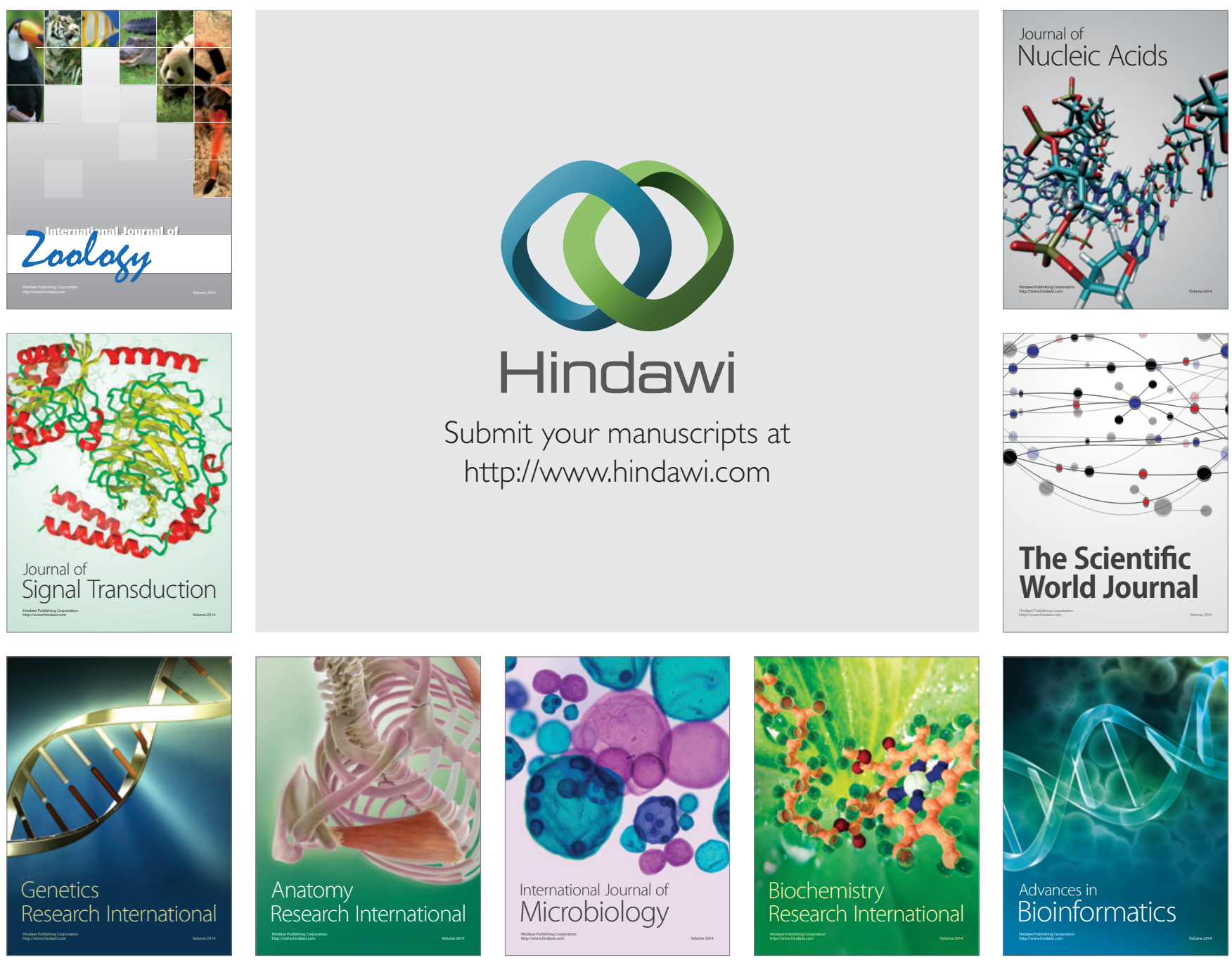

The Scientific World Journal
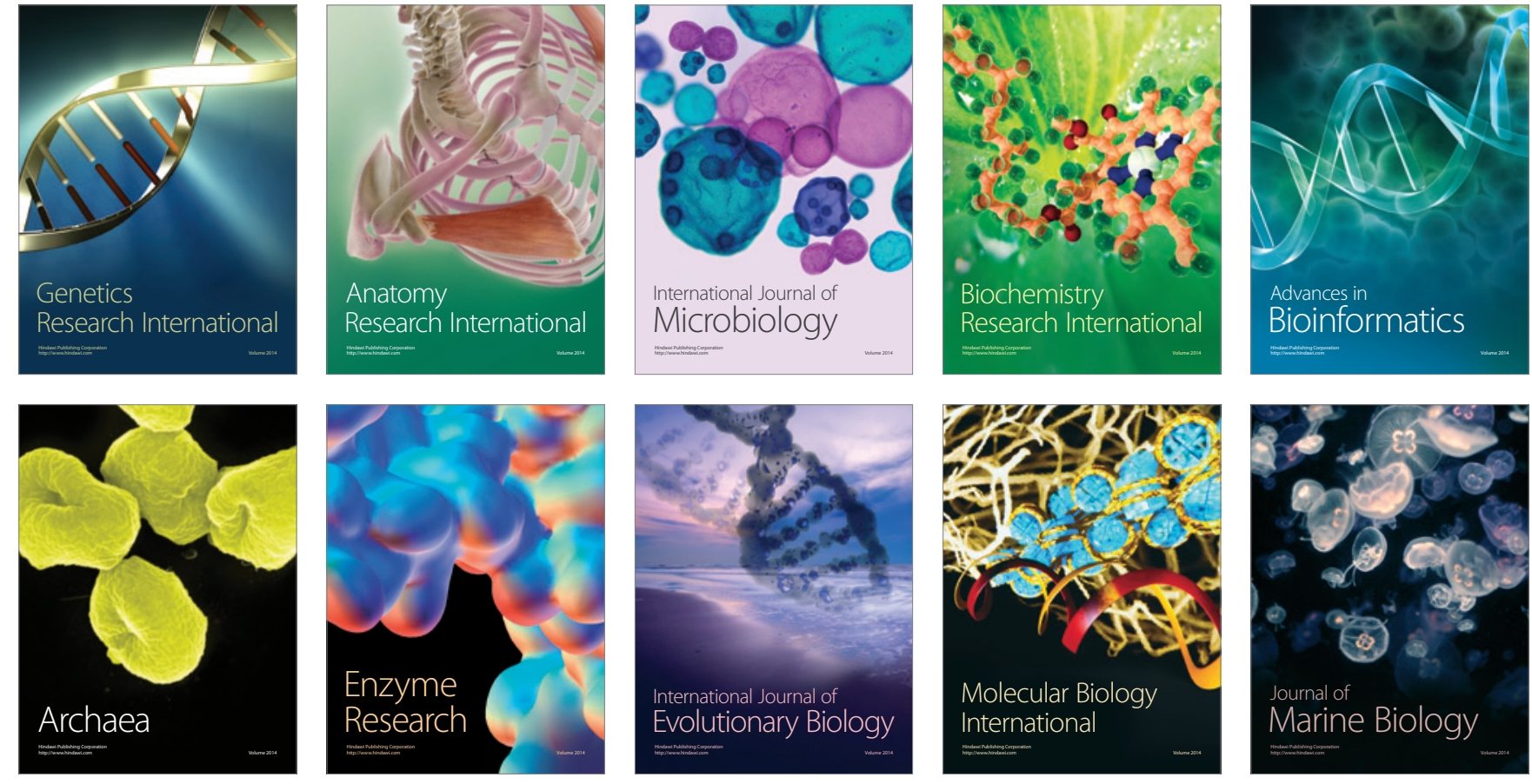